\title{
Applying Salutogenesis in Healthcare Settings
}

\author{
Jürgen M. Pelikan
}

\section{The Challenge of Integrating Salutogenesis into Health Care}

Health care, or more correctly the "disease care system" (Antonovsky, 1996, p. 12), is a very specific and challenging area for applying salutogenesis. And for health or disease care, implementation of salutogenesis is quite a challenge as well.

What is the essence of these challenges of integrating these two health-related fields? The healthcare sector still primarily follows a pathogenic paradigm. It intends to professionally manage illness by trying to cure, what is defined as a disease, or, if this is not possible, at least to offer care for chronic patients and palliative care. But the contribution of health care to public health, or health promotion more specifically, is still marginal. Reorientation of health services, as demanded by the Ottawa Charter (World Health Organization, 1986), has not happened to a remarkable degree yet (De Leeuw, 2009; Wise \& Nutbeam, 2007). There still is quite an unrealized potential in health care to be more preventive of disease and more protective and promotive of positive health.

Salutogenesis as defined by Antonovsky has been developed as a paradigm in opposition to this "pathogenic orientation which suffuses all western medical thinking" (Antonovsky, 1996, p. 13). Therefore, in principle, applying salutogenesis to health care means to restrict the leading pathogenic orientation in healthcare practice (research and policy) and complement or change it by a salutogenic orientation in everyday practice and research. This can only partly be established as an add-on of new routines, and partly has to be done as an add-in to ongoing practices, by reorienting core processes of health care.

J. M. Pelikan ( $₫)$

Institute for Sociology, University of Vienna, Vienna, Austria

WHO-Collaborating Centre for Health Promotion in Hospitals and Healthcare at the Austrian National Public Health Institute

(Gesundheit Österreich GmbH), Vienna, Austria

e-mail: juergen.pelikan@univie.ac.at
As health care and its quality discourse are dominated more and more by the dictum of evidence-based practice, if salutogenesis is to be acceptable in health care, it has to demonstrate its evidence-based character. But salutogenesis, a partly normative concept, also has quite an unrealized potential for being more evidence-based. Antonovsky himself stated, "in short, at the present time, the appeal of the full salutogenic model for those engaged in health promotion cannot be on the grounds of powerfully demonstrated efficacy in producing significant health-related change outcomes" (ibid., 16). The relevant question, therefore, is how far has this changed since Antonovsky wrote this statement?

Salutogenesis - the newer and more focused concepthas been introduced by Antonovsky into health promotion, an older and broader concept, field, and movement. As Antonovsky saw it, "the basic flaw of the field (of health promotion) is that it has no theory." And he proposed "the salutogenic orientation... as providing a direction and focus to this field." But he also believed, "the salutogenic model is useful for all fields of health care. In its very spirit, however, it is particularly appropriate to health promotion" (ibid., 18). Thus, health promotion in health care definitely has the blessings of Antonovsky. Therefore, we have to clarify how the salutogenic orientation or model and its related construct of sense of coherence (SOC) can be integrated into health care, directly or via (re-)orienting health promotion in health care indirectly.

\section{What Does Salutogenesis Specifically Mean for Health Care?}

In health care, the salutogenesis paradigm can be used in principle for two purposes: either to guide health promotion interventions in healthcare practice or to (re)orient healthcare research. For this, the salutogenesis paradigm offers specific concepts, assumptions, and instruments. Three quite different conceptual forms can be distinguished: a salutogenic orientation, a salutogenic model, and the construct of 
the SOC and a "methodologically respectable way to operationalize it" (ibid., 13) by an instrument to measure it. These three forms first have to be specified in more detail, to be applied later to the field of health care. For that, health care has to be understood as a complex of a strongly interrelated professional practice, with clinical research and supporting policy. Therefore, applying salutogenesis in health care successfully cannot just be done by introducing salutogenesis in healthcare practice, but must also include salutogenic clinical research, and change in underlying healthcare policy.

The first and most broad form of salutogenesis, a salutogenic orientation, is described by three assumptions:

- "That the human system (as all living systems) is inherently flawed, subject to unavoidable entropic processes and unavoidable final death" (ibid., 13). Therefore, the necessity of adaptation or coping with accompanying tension that may result in stress is universal and not the exemption.

- "A continuum model, which sees each of us, at a given point in time, somewhere along a healthy/dis-ease continuum" (ibid., 14). Therefore, a dichotomization of people into healthy and sick is arbitrary and not adequate.

- The concept of salutary factors (or health-promoting factors): "factors which are negentropic, actively promote health, rather than just being low on risk factors" (Antonovsky, 1996, p. 14). Therefore, risk and salutary factors have to be attended to.

From these three assumptions follows implications for health promotion:

"A salutogenic orientation, as the basis for health promotion, directs both research and action efforts

- To encompass all persons, wherever they are on the continuum.

- And to focus on salutary factors" (ibid., 14).

- This "must relate to all aspects of the person" (ibid., 14) instead of "to focus on a particular diagnostic category" as in curative medicine or also in preventive medicine, that is, to include primary prevention or secondary prevention (ibid., 14).

Applying these assumptions and implications to healthcare practice would mean that:

1. Since a salutogenic orientation encompasses all persons independent of their position on the healthy/disease continuum, health care not only should just care for the health of its patients but also has to take responsibility for the health of its staff and the health of citizens in the catchment area as well (while dichotomous classification of persons into those who show indications for some spe- cific disease or not seems to be still unavoidable for doing diagnosis-oriented curative medicine on patients).

2. In relation to these three groups of stakeholders, not only their risk factors have to be dealt with or fought by health care but also possible salutary factors have to be enhanced as well in curative, preventive, protective, and promotive practice.

3. A holistic approach, including physical, mental, and social dimensions, respectively, ill and healthy aspects of a person, has to be taken into account in dealing with all people affected by health care.

In principle, to apply these demands on health care sounds plausible and rational. But, to realize, (1) a policy change of the mandate of health care is necessary, (2) the traditional diagnostic and therapeutic repertoire of health care has to be widened, and (3) a radical change of clinical outlook is implied. The last reorientation is especially difficult, since part of the spectacular medical success rests on focusing on a narrow biomedical model.

The second form of salutogenesis is Antonovsky's specific and rather complex salutogenic model (described in Chap. 7 of Antonovsky, 1979). Within this model, the concept of generalized resistance resources (GRRs) is introduced as "a property of a person, a collective or a situation which, as evidence or logic has indicated, facilitated successful coping with the inherent stressors of human existence" (Antonovsky, 1996, p. 15). Major psychosocial, genetic, and constitutional GRRs are specified within this model. But this model has not much been taken up by Antonovsky or other authors in later publications (Mittelmark \& Bull, 2013).

When using this salutogenic model in health care, the generalized resistance resources specified in detail in the model would have to be more adequately taken into account in healthcare practice and research. This makes much sense for healthcare and affords a more holistic and complex outlook and a widening of diagnostic and therapeutic methods applied.

The third most focused form of salutogenesis, the specific construct of sense of coherence (SOC), which has been introduced as a central factor in the salutogenic model of health, is defined as follows:

a generalized orientation toward the world which perceives it, on
a continuum, as comprehensible, manageable and meaningful.
The strength of one's SOC, I proposed, was a significant factor
in facilitating the movement toward health." This construct
answers "what do all these GRRs have in common, why do they
seem to work. What united them, it seemed to me, was that they
all fostered repeated life experiences which, to put it at its sim-
plest, helped one to see the world as 'making sense,' cognitively,
instrumentally and emotionally. (Antonovsky, 1996, p. 15)

One way to interpret this is that Antonovsky introduces the SOC rather as a moderator or mediator of other 
determinants of health than as a specific further determinant of health. Compared to other familiar concepts from the coping literature, "it is the particular combination of the cognitive, behavioral and motivational which is unique" and, furthermore, "the SOC is not a culture-bound construct." "What matters is that one has had the life experiences which lead to a strong SOC; this in turn allows one to 'reach out,' in any given situation, and apply the resources appropriate to that stressor." "The strength of one's SOC (as a dependent variable) is shaped by three kinds of life experiences: consistency, underload-overload balance, and participation in socially valued decision-making. The extent of such experiences is molded by one's position in the social structure and by one's culture..." (ibid., 15). Two instruments/tools have been offered to measure the SOC, a longer 29-item SOC scale and a shorter 13-item version, but both are not suitable to measure the three specific subdimensions of the SOC (Antonovsky, 1993).

How can the SOC be introduced into health care? Being ill and becoming a patient in professional healthcare services often is a rather threatening life experience for people and being a healthcare professional is a rather demanding kind of job. Therefore, using the SOC concept for making healthcare structure and culture as far as possible consistent, underloadoverload balance and participatory for patients, staff, visitors and citizens generally would be an adequate and welcome application to make healthcare systems and services more salutogenic, generally. This is possible, since "social institutions in all but the most chaotic historical situations can be modified to some degree" (Antonovsky, 1996, p. 15). It even could be more feasible, effective, and efficient to develop salutogenic "standards" (Dalton \& Mccartney, 2011) and make institutional contexts more salutogenic, than to try to directly enhance the SOC of large numbers of patients, staff, and citizens. Thus, patients and staff could be supported by healthcare organizations to experience their respective roles and tasks as comprehensible, manageable, and meaningful to allow for successful co-production in actual cure and care and of better health in the long run. That, at least, would reduce avoidable stress, most important for people with a low SOC. More specifically, the SOC of patients or staff could be measured or screened, and their level of SOC be taken into account in treating or deploying them, even if this seems to be a rather far reached and also problematic stigmatizing kind of application. Even if Antonovsky assumed that one's SOC could not be radically transformed, he left it open that the SOC could "be shaped and manipulated so that it in turn can push people toward health" (Antonovsky, 1996, p. 15). Therefore, improving one's SOC or at least one's health literacy could become an explicit goal of (chronic) disease management.
In summary, salutogenic thinking has good potential to be applied to health care in relation to health-promoting interventions for the health of patients, staff, and citizens, and in supporting health-promoting structures and cultures of healthcare institutions for better everyday practice and policy.

\section{Overview of Chap. 37, the Application of Salutogenesis in Hospitals, by Christina Dietscher, Ulrike Winter, and Jürgen M. Pelikan}

This chapter is reprinted from the 2017 edition. Hospitals, in developed countries the center of curative health care in practice, research, and education, still have a dominantly pathogenic orientation. Therefore, salutogenic principles definitely have to offer quality improvement of cure and care in hospitals. But salutogenesis also is a considerable challenge to be implemented in hospitals, and hospitals are challenging for health and salutogenesis promoters. Chapter 37 first demonstrates how salutogenesis, if understood as a specific dimension of hospital quality, could considerably contribute to better health gain for patients and hospital staff. Second, drawing on a comprehensive literature search, it is highlighted which aspects of salutogenesis in relation to hospitals already are covered in descriptive and intervention research focusing on patients (and family members), staff, and the hospital as an organization. Topics included are concepts of salutogenesis referred to, the SOC in relation to physical symptoms; the SOC in relation to mental symptoms, quality of life, and patient satisfaction; the SOC adjustment to disease, self-management, and adherence to treatment; the SOC and social outcomes; the SOC and positive health; the SOC in relation to gender, age, and socioeconomic status; the SOC in relation to patients' family members; salutogenesis in general and the salutogenic model; salutogenesis and impacts of the hospital setting on patients; using the SOC as a diagnostic tool; adapting treatment schemes; supporting self-care and self-management; supporting caring relatives; improving the impact of hospital functioning on salutogenesis; salutogenesis for different healthcare professions; and implications for occupational health in hospitals.

An overview of the application of salutogenesis in healthpromoting hospitals, one of the WHO-initiated settingoriented health promotion networks, also is provided.

Needs for further research are outlined focusing mainly on the specific role of the sense of coherence as a predictor, mediator, or moderator, by better conceptual clarity and more complex research designs; on the interlink between the SOC and other aspects of health than subjective and mental health; 
on the impact of hospital functioning and organizational interventions on salutogenesis or the SOC specifically; and on the applicability of the SOC as a measurement to assess the outcome of health promotion interventions in hospitals.

\section{Overview of Chap. 38, Applying Salutogenesis in Primary Health Care, by Daniela Rojatz, Peter Nowak, Ottomar Bahrs, and Jürgen M. Pelikan}

Primary care is understood in Chap. 38 as the first contact point to medical care. It operates at the interface between the social and the health systems, between the patient with his or her family and the professional environment, and refers to the local population, while primary health care, following WHO, is defined as a whole-of-society approach envisioned to contribute to universal health coverage and equality. This chapter is dedicated primarily to the application of salutogenesis in primary care. Since primary care services are a complex of strongly interrelated professional practice, research, and supporting policy, applying salutogenesis in primary care comprehensively should introduce salutogenesis in all these fields simultaneously.

This chapter examines how salutogenesis is addressed and discussed in policy, research, and practice of primary care and discusses the application of salutogenesis as an orientation, a model, and the construct of "sense of coherence." Thus, it contributes to supporting the application of salutogenesis in primary care and provides an outlook on further research needs.

In a first step, the chapter tries to make comprehensible what characterizes primary care, salutogenesis, and, finally, the meaningfulness of salutogenic primary care. Subsequently, the chapter examines how salutogenesis is addressed and "managed" in policy, research, and practice of primary care. Moreover, it discusses the application of salutogenesis as a health orientation, model, and sense of coherence in primary care. Concerning policy, relating salutogenic primary care to public health and health promotion is outlined, by focusing on relevant documents by WHO and the UN Sustainable Development Goals. For research, a literature review was conducted and results were presented in relation to the use of salutogenesis as an orientation, a model, or the SOC. For practice, two pilot projects from Austria were described, one focusing on the use of the concept of "review dialogue" in GP practices in Germany and the other on developing a comprehensive primary care concept within the Austrian healthcare reform, taking explicitly into account the macro-, meso- and micro-level of primary care services. Finally, lessons for the implementation of salutogenic primary care are considered, and more detailed recommendations are summarized in the concluding part of the chapter.

\section{Overview of Chap. 39, Applying Salutogenesis in Mental Healthcare Settings, by Eva Langeland and Hege Forbech Vinje}

Chapter 39, which is a revised version of a chapter in the 2017 edition, deals with salutogenesis for a specific and growing group of patients. It emphasizes the importance of high-quality social support in interplay with positive identity development. Antonovsky's core concept of sense of coherence has been shown to be more closely related to mental health than to physical health. Thus, the application of salutogenesis on patients in mental healthcare settings is rather obvious. First, this holds for the principal paradigmatic understanding of mental health problems or disorders as a challenge for patients which depends on the individual's personal way of experiencing it, by their healthcare professionals. Second, it can result in specific forms of salutogenic therapy, for example, talk therapy groups that aim to support positive salutogenic identity building as a specific resistance resource and to improve the sense of coherence of patients by specific offers of social support. This approach emphasizes increasing participants' awareness of and confidence in their potential internal and external resources and possibilities to cope successfully and effectively manage tension. Third, as in all health care, the material and social setting itself should be designed by salutogenic principles as empowering by being comprehensible, meaningful, and manageable. This is especially important for more sensitive and vulnerable chronic mental patients who also experience longer stay in mental healthcare organizations.

Some experimental evidence for the feasibility and effectiveness of this kind of therapy is offered, while systematic intervention research on this application of salutogenesis in mental healthcare is still rather limited.

\section{Overview of Chap. 40, Applying Salutogenesis in Vocational Rehabilitation Settings, by Monica Lillefjell, Ruca Maass, and Camilla Ihlebæk}

In Chap. 40, which is a revised version of a chapter in the 2017 edition, rehabilitation services are more closely and directly linked to maintaining and regaining positive health lost by illness and by pathogenic side effects of health care than the provision of cure or care. There even exists some professional understanding that rehabilitation should start with the beginning of treatment and be integrated into treatment processes and not just follow after discharge of patients. But even the WHO definition of rehabilitation has a pathogenic bias by focusing on disabilities of people or on disabled people and not addressing their abilities explicitly, even if rehabilitation is defined as enabling "for optimal 
physical, sensory, intellectual, psychological and social functioning." Therefore, salutogenesis still has to offer something and has an added value to rehabilitation as a supportive intervention for recovery processes. In addition, rehabilitation itself can be seen as a process where participants have to deal with considerable challenges at biological, psychological, and social levels, and their coping will be influenced by the existing level of the participants' sense of coherence.

Within the wider field of rehabilitation, this chapter has a specific focus on vocational work-oriented rehabilitation which is a combination of medical, psychological, social, and occupational activities with the goal of enabling a timely return to work after sickness absence. For that, the chapter highlights how salutogenesis can be related to the design and implementation of vocational rehabilitative services. A summary of descriptive and intervention research is given on the impact of the SOC as a moderator on processes and outcomes of rehabilitation programs and on the influence of these programs on the development of the SOC, which shows that there is empirical evidence for both kinds of effects. Recommendations for further research with more complex longitudinal designs are given, but the greatest challenge in the future will be not only just strengthening individuals by salutogenic rehabilitation programs but also assessing and influencing problematic challenges of workplace environments by these programs.

\section{Overview of Chap. 41, Applying Salutogenesis in Residential Care Settings, by Viktoria Quehenberger and Karl Krajic}

Chapter 41, a revised version of a chapter in the 2017 edition, focuses on aged and highly aged patients who have long and rather comprehensive contacts with healthcare institutions of long-term care, either in residential aged care or in community dwelling. Therefore, it is well accepted in the literature that a salutogenic orientation and health promotion measures could contribute to the quality of life, well-being, and health of this group. Furthermore, a good sense of coherence can be considered as a positive resource for coping with the physical, mental, and social challenges and transitions related to aging.

But the state of descriptive research on salutogenesis focusing not only on residents but also somewhat less so on community dwellers is still scarce and has mostly been conducted in few countries. Different subjective and objective health outcome measures have been used on the two groups, but scarcely more complex theoretical assumptions have been researched. There is research on determining, mediating, or moderating effects of the SOC on health outcomes, but results are still diverse. There also exist studies exploring the stability of sense of coherence in older age, but due to their cross-sectional design, their results have to be interpreted with caution.

Concerning intervention research, only "very few studies have specifically applied salutogenic principles to promote positive health among older people." Mostly studied were consequences of physical activity interventions which had positive effects on sense of coherence and well-being indicators. One study also showed an increase in the sense of coherence by psychotherapy.

In light of this scarce research situation, the authors make recommendations for further research in this relevant and growing area of health care which should make use of better clarified theoretical assumptions and hypotheses with more complex comparative cross-sectional or even better longitudinal designs and more elaborated measures for GRRs and SOC. Furthermore, it has to be dealt with one of the major limitations of existing research, where aged and highly aged with cognitive impairment have mostly been excluded from the research.

\section{Overview of Chap. 42, Applying Salutogenesis in Midwifery Practice, by Sally Muggleton and Deborah Davis}

Chapter 42 takes up midwifery as unique among the healthcare professions because it mostly focuses on physiological processes and a period of transition in the life of a woman and her family. Thus, midwives work across a childbearing continuum and the health-ease dis-ease continuum. While the autonomous scope of midwifery practice lies in uncomplicated childbirth, midwives also have an important part to play in the care of women with health complexities in close collaboration with medical or other colleagues. The "midwifery model of care" and its approach to childbearing focus on wellness rather than illness and work closely with women to help them mobilize their own resources to move toward greater health. But the contrasting pathogenic approach to maternity care is still ubiquitous in contemporary healthcare provision with over-medicalization of childbirth and overuse of interventions, which can also cause more harm than good.

While there is resonance between midwifery practice and salutogenesis, research examining the relationship is still in its infancy. Few researchers explicitly draw on salutogenic theory. Of these, few studies and scoping reviews are described in more detail. They suggest that there is an alignment between salutogenesis and midwifery practice. A number of studies found that women with a strong sense of coherence had improved emotional health, are more likely to engage in healthy behaviors and seek useful support. These women are also more likely to experience uncomplicated birth, birth at home and identify normal birth as their preferred mode of birth. A salutary childbirth education 
program, based on these findings, was established, piloted, and evaluated by the authors of the chapter and is described in more detail. This program explicitly uses key concepts of salutogenesis.

The chapter concludes by stressing that salutogenesis, with its focus on health rather than pathology, offers a promising way forward to underline that much of the work of midwives' care for women is health promotion but has to be operationalized accordingly in midwifery practice. An overview of the project on salutary childbirth education focusing on generalized resistance resources and increasing individual sense of coherence by strengthening its key components of comprehensibility, manageability, and meaningfulness has been provided. A next step could be to extend the salutogenic approach beyond childbirth to maternity services in general.

\section{Overview of Chap. 43, the Application of Salutogenesis in Birth, Neonatal, and Infant Care Settings, by Soo Downe, Shefaly Shorey, Claudia Meier Magistretti, and Bengt Lindström}

Chapter 43 starts with a short summary highlighting Antonovsky's unique contribution to theory, practice, and policy concerning the origins and experience of health, as well as the causes and manifestations of disease. The relation of salutogenesis to maternity care is discussed by giving a critical overview of studies in perinatal care, primarily measuring and promoting parental SOC and well-being. A turn toward a positive experience is observed, driven by a new emphasis on finding out what matters to women, families, service providers, and policymakers, based on qualitative evidence of their views and experiences. Based on this, maternal health-promoting interventions have been strongly recommended by WHO in recent guidelines.

Next, an overview is given on salutogenic approaches to neonatal and infant service provision. The dependency of neonatal well-being on what has happened in utero and during birth is highlighted. Skin-to-skin contact, breastfeeding, and family-centered care in hospitals are examined as important aspects of and salutogenic interventions for parent-child attachment in the first year of a child's life. Infancy and early childhood are critical developmental periods for language and communication skills, cognitive skills, socioemotional functioning, and self-regulation. Therefore, parents' and caregivers' relationship with their infants and newborns plays a critical role in shaping the emotional, cognitive, and social development of their child. Thus, secure attachments have been associated with strong SOC expression. Different interventions of early support to optimize parenting capacity and their impact are discussed next, including strengthening the SOC in families and access to early education offers, based on a large-scale evaluation study in Switzerland. Special attention is given to the importance of coherence in early support.

In the discussion part of the chapter, it is stressed that only examples of salutogenic approaches based on reasonable evidence could be described in this chapter. But there is a growing awareness of the value of salutogenic approaches to the provision of maternity care and to facilities and services to enhance parenting and well-being in infancy and early childhood. Research gaps are identified, and suggestions for the direction of future research are outlined. The chapter concludes by stating that salutogenic theory is as relevant for describing and catalyzing maternity, parenting, and infant well-being as it is for other phases of human life.

\section{Overview of Chap. 44, Applying Salutogenesis in Community-Wide Mental Health Promotion, by Vibeke Koushede and Robert Donovan}

Chapter 44 first highlights the relevance of mental health as a resource and risk for population health and describes mental health problems and related financial and social implications for society, which has led to an increased focus on prevention of mental health problems in health policy lately. Using the river metaphor of salutogenesis and a mental health ease-disease continuum, mental health is seen not as a stable trait but rather as a constant process, which needs to be protected and promoted. Thus, mental health promotion is foremost focused on protective factors and promoting mental health resources at different levels of society and is relevant to everyone.

Second, the "Act-Belong-Commit"/"ABCs of Mental Health" Campaign is described as a world-first comprehensive, population-wide, community-based mental health promotion campaign designed to promote mental health and prevent mental ill-health, based on a grounded theory model. This campaign, which is consistent with the salutogenic approach of aiming to build positive mental health rather than targeting specific risk factors for specific mental illnesses, originated in Western Australia and is now diffusing nationally and internationally. This campaign developed a specific ABC message, act, belong, and commit, which was implemented in Western Australia using a community-based social marketing approach. The campaign also has been operated in Denmark, where the Act-Belong-Commit framework has been adapted first and then implemented.

In summary, the chapters of this part demonstrate for different services of health or disease care that applying the salutogenic orientation, the salutogenic model and the concept, and instrument of the SOC has the potential to improve the health-promoting quality of structures, processes, and 
outcomes of these services for enhancing health gain and well-being of different stakeholders. There already exists impressive research-based evidence for this, but also quite a potential for further more systematic and complex research.

\section{References}

Antonovsky, A. (1979). Health, stress and coping. Jossey-Bass.

Antonovsky, A. (1993). The structure and properties of the sense of coherence scale. Social Science \& Medicine, 36(6), 725-733.

Antonovsky, A. (1996). The salutogenic model as a theory to guide health promotion. Health Promotion International, 11(1), 11-18.
Dalton, C., \& Mccartney, K. (2011, May 23-26). Salutogenesis: A new paradigm for pervasive computing in healthcare environments? Conference: 5 th international conference on pervasive computing Technologies for Healthcare, pervasive health 2011, Dublin. doi: https://doi.org/10.4108/icst.pervasivehealth.2011.246064.

De Leeuw, E. (2009). Have the health services reoriented at all? Health Promotion International, 24(2), 105-107.

Mittelmark, M. B., \& Bull, T. (2013). The salutogenic model of health in health promotion research. Global Health Promotion, 20(2), 30-38.

Wise, M., \& Nutbeam, D. (2007). Enabling health systems transformation: What progress has been made in reorienting health services? Promotion and Education, 2, 23-27.

World Health Organization. (1986). Ottawa charter for health promotion: Towards a new public health. World Health Organization.

Open Access This chapter is licensed under the terms of the Creative Commons Attribution 4.0 International License (http://creativecommons. $\mathrm{org} / \mathrm{licenses} / \mathrm{by} / 4.0 /)$, which permits use, sharing, adaptation, distribution and reproduction in any medium or format, as long as you give appropriate credit to the original author(s) and the source, provide a link to the Creative Commons license and indicate if changes were made.

The images or other third party material in this chapter are included in the chapter's Creative Commons license, unless indicated otherwise in a credit line to the material. If material is not included in the chapter's Creative Commons license and your intended use is not permitted by statutory regulation or exceeds the permitted use, you will need to obtain permission directly from the copyright holder. 